\title{
The Optimal Focus Position When Scrolling Using a Small Display
}

\author{
James Whalley and Andrew Monk \\ CUHTec, Department of Psychology, University of York, \\ York, YO10 5DD, UK \\ A.Monk@psych.york.ac.uk \\ http: / /www. cuhtec.org.uk/
}

\begin{abstract}
When scrolling through a list on a small display, such as that on a cell phone, the "focus" is the currently highlighted item that would be selected were the user to stop scrolling and choose select. When scrolling through a list that is longer than the number of items that may be displayed simultaneously, the focus position becomes stationary and the items scroll under it. An experiment is reported which varies this stationary focus position in a five item display. It was either: the last item in window (end stop scheme), the next to last (view forward one item) or the centre (view forward two items). The centre position allowed significantly faster scrolling than the other two positions.
\end{abstract}

\section{Introduction}

Scrolling is the term used to describe various interaction techniques for varying the window of displayed material onto a sequential object such as a document or list that is too large to be displayed all at once. For example, there are well established conventions for using a scroll bar in graphical user interfaces utilising a mouse or stylus. This paper is concerned with small devices such as cell phones where conventions for scrolling are still developing.

With these very small digital displays there is often no pointing device to parallel the mouse and hence no way to select an item with a single action. The alternative is to display a "focus". This is the item that is visually highlighted as being the one that would be selected were the user to choose "select". In the first panel of Fig. 1, item 3 is under the focus in the centre of the display. Subsequent panels in Fig. 1 show how, initially, the focus moves down the display as the user scrolls. When the focus reaches the bottom of the window it remains stationary and the list moves under it (see last two panels in Fig. 1). This "end stop" scheme is commonly used on cell phones. Fig. 2 depicts a small variation on this interaction technique to provide "view forward" which is found on some phones. Here the focus becomes stationary and the list starts to move when the focus reaches the penultimate item in the display. This provides the user with a fleeting opportunity to see an item before it becomes the focus. Still other phones keep the focus stationary at the centre of the display. This paper reports an experiment which varies the focus position in a five item display: last item visible, next to last and centre. The aim was to identify the optimal focus position for rapid scrolling through a large list such as an address list. 


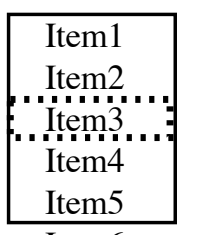

Item6

Item7

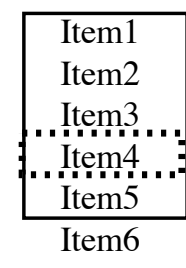

Item7
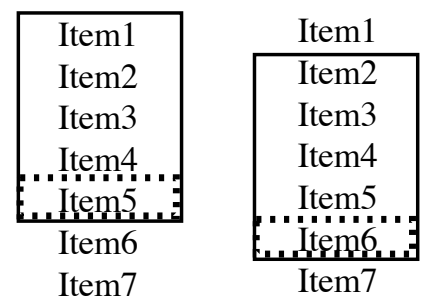

Fig. 1. Standard scrolling scheme (end stop), the solid box is the window displayed, the dotted box is the focus

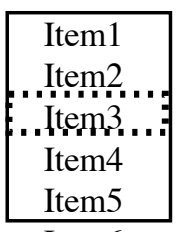

Item6

Item7
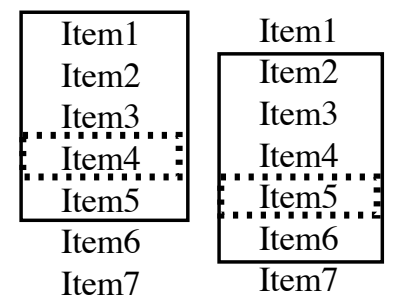

Item1

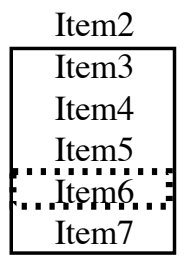

Fig. 2. View forward scheme, see Fig. 1 for explanation

\section{Method}

A 50 item list was constructed consisting of randomly paired first and second names. These were presented at 1.5, 2.5, 3.5, and 4.5 items per second on a small display simulated on a PC. Pressing the mouse button commenced scrolling, letting it go stopped it. On each trial the user was given a target name and asked to stop scrolling with this target in focus. Any trial when they did this on the first press was counted as a hit.

As suggested above, analysis of the user's task suggested that focus positions 3 and 4 might have advantages over position 5 because the target could be seen before it came under the focus. This advantage would be expected to be most evident when the user has no knowledge of what is coming next in the list. This was the case in the randomly presented lists, where the list order was randomised separately for each trial. However, this is not very natural. An address list, for example, will generally be alphabetised and hence always appear in the same order. For this reason half the trials used a list presented in the same alphabetical order each time.

Six experimental conditions were formed by factorially combining the three focus positions with the two list orders. With 20 replications this means each of the 6 users completed 480 trials. Testing was split into 120 trial blocks. Within each block there were 10 replications of each Focus Position by Speed of Scrolling condition randomised separately for each participant. Two blocks (one alphabetic and one random) would be completed on one day with a half-hour gap between, with the remaining two blocks a week later, The order participants received the blocks was counterbalanced between days and participants. The 6 participants in this experiment were all undergraduate students. 


\section{Results}

The raw data from this experiment can be visualised as a plot of hit rate against speed of presentation for each combination of List Order and Focus Position. These plots have the classic ' $\mathrm{S}$ ' shape of a psychophysical function. At the slower speeds hit rates approach $100 \%$ and at the faster rates they approach $0 \%$. For each participant curve fitting (a cumulative normal distribution) was used to interpolate the speed that would have resulted in a hit rate of $75 \%$. This was taken as the maximum acceptable scrolling speed for that experimental condition. These rates in items per second were then submitted to analysis of variance. Means items per second for the three Scrolling Positions and the two List Orders are presented in Fig. 3.

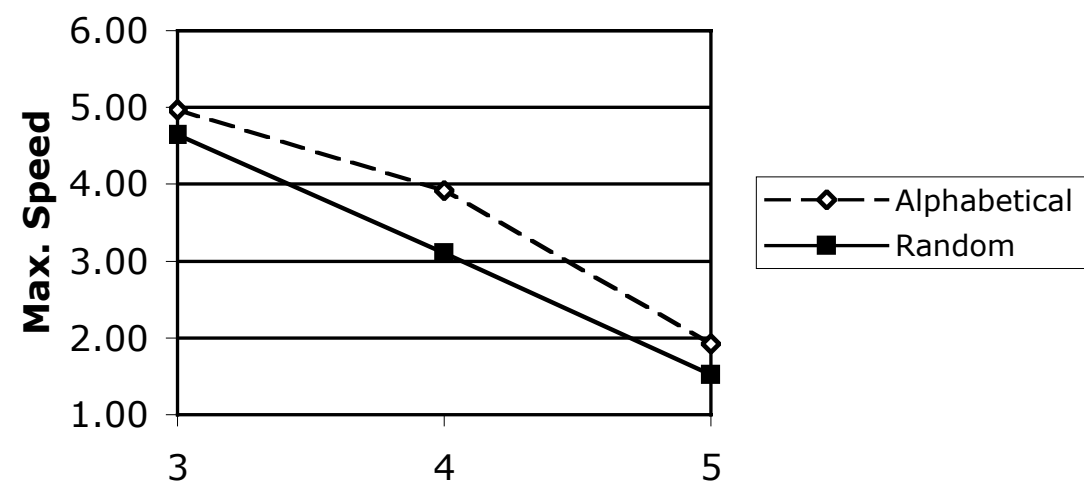

Fig. 3. Mean interpolated maximum speed in items per second for random and alphabetical lists. Position 3 is the centre of the display, and position 5 is the last.

These results can be straightforwardly interpreted as a main effect of Focus Position $(\mathrm{F}(2,10)=44.513, \mathrm{p}<.05)$ and a smaller main effect of List Order $(\mathrm{F}(1,10)=$ $18.313, \mathrm{p}<.05)$. There was no significant interaction, i.e., the effect of focus position is the same for both list orders $(\mathrm{F}(2,10)=1.501$, n.s $)$. The effect of focus position is considerable, moving it up from position 5 to position 4 doubles the speed at which users can scroll. There was a similar increase in the estimated maximum speed moving from position 4 to position 3. Tukey's HSD test showed that all pairwise comparisons of these three means were statistically significant.

\section{Implications for Design}

Users can scroll faster when they have preview of the items before they fall under the cursor and having preview of two items permits faster scrolling speeds than having one. On this basis it is recommended that the focus position should be at least two items up, and not the last item in the window as is commonly the case with cell phones. 
It is not clear how this result arises. It could be because users are then able to fixate the bottom line of the display, allowing for reaction time when the target comes in view. If this were the case, then one might expect it to be reflected in the rates expressed as seconds per item rather than items per second. In fact, the difference between two items preview (centre position) and one item (penultimate) is only .077 s/item as compared with $.296 \mathrm{~s} /$ item for the difference between no preview (last) and one item. Alternatively the users may be fixating the focus and extracting information about the up coming items in peripheral vision. The results of Eriksen and Eriksen (1974) indicate that this is possible with the display used here. The likely answer is that there is some complex combination of peripheral vision and fixation strategy going on here that can only be properly explored with eye movement monitoring.

Five items per second is faster than the average phone currently scrolls. Of course there will be large differences in acceptable scrolling rates across individual users and so to take advantage of the faster rates that are possible the user needs to be given control of the scrolling rate. How this may is done depends very much on the hardware controls available. An isometric joystick where pressure maps onto speed of scrolling would be very advantageous and could potentially make the phone a Fitts' Law device (MacKenzie, 2003). Thus, when scrolling an alphabetised list the user could use knowledge of the approximate position of the target item to vary the speed of scrolling efficiently.

Of course, there are other factors than scrolling speed governing the position of the cursor such as ease of learning and ease of use. The next step is to test such a scheme with new and experienced users and using real phones to check there are no unforeseen side effects of making such a change.

\section{References}

1. Eriksen, B.A. and Eriksen, C.W. (1974) Effects of noise letters upon the identification of a target letter in a nonsearch task. Perception and Psychophysics, 16, 143-149.

2. MacKenzie, I.S. (2003) Motor behaviour models for human-computer interaction, in Carroll, J.M. (Ed.) HCI Models, theories and frameworks, San Francisco: Elsevier Science, pp27-54. 